\title{
Erratum
}

\section{A Finite Sum Representation of the Angular Momentum Projection Operator}

P. Chattopadhyay

Z. Physik A 292, 61-65 (1979)

The right hand sides of Eqs. (2.12), (2.17) and (3.2) should be multiplied by $\frac{1}{(2 M+1)^{3}}, \frac{1}{(M+1)^{2}(2 M+1)}$ and $\frac{1}{(2 M+1)}$ respectively. Similarly a factor of $\frac{1}{(M+1)}$ is missing from the r.h.s. of Eqs. (3.6) and (3.10).

In Eq. (2.17) the factor $e^{-i \beta_{0}^{\prime} l \hat{J}_{y}}$ should be replaced by $e^{-i \beta_{0} l \hat{J}_{y^{\prime}}}$ and the expression of $A_{K K^{\prime}}^{n l n^{\prime}}\left(J, \beta_{0}\right)$ appropriately modified.

In (A.9) the phase factor is $(-1)^{K-K^{\prime}}$ instead of $(-i)^{K-K^{\prime}}$. The power of $\left(\cos \frac{1}{2} \beta\right)$ in $(A .15)$ is $\left(K+K^{\prime}\right)$.

The part between (A.10) and (A.14) in the Appendix should read as follows:

The integrals are different depending on whether $(l-m)$ is odd or even. Denoting by $I_{K K^{\prime}}^{0}(J, m)$ and $I_{K K^{\prime}}^{E}(J, m)$ the parts of the sum such that $(l-m)$ is odd and even respectively one has

$$
\begin{aligned}
& I_{K K^{\prime}}^{0}(J, m)=\sum_{l=0}^{J} \frac{(-i)^{K-K^{\prime}} d_{l K^{\prime}}^{J}\left(\frac{1}{2} \pi\right) d_{l K}^{J}\left(\frac{1}{2} \pi\right)}{2 i} \\
& {\left[\delta_{l, m-1}-\delta_{l, m+1}\right] \pi} \\
& =\frac{\pi}{2 i}(-i)^{K-K^{\prime}}\left[d_{m-1, K^{\prime}}^{J}\left(\frac{1}{2} \pi\right) d_{m-1, K}^{J}\left(\frac{1}{2} \pi\right)\right. \\
& \left.-d_{m+1, K^{\prime}}^{J}\left(\frac{1}{2} \pi\right) d_{m+1, K}^{J}\left(\frac{1}{2} \pi\right)\right] .
\end{aligned}
$$

and

$I_{K K^{\prime}}^{E}(J, m)=-2(-i)^{K-K^{\prime}} \sum_{l=0}^{J}{ }^{\prime \prime} \frac{d_{l K^{\prime}}^{J}\left(\frac{1}{2} \pi\right) d_{l K}^{J}\left(\frac{1}{2} \pi\right)}{(l-m)^{2}-1}$

where $\sum^{\prime}$ and $\sum^{\prime \prime}$ indicate the appropriate restrictions on the summation. One can show that the real part of $I_{K K^{\prime}}(J, m)$

$I_{K K^{\prime}}^{R}(J, m)=0|m|<\left(J-K_{1}\right)$

where $K_{1}$ is the greater of $K$ and $K^{\prime}$. Thus since the $d$-functions are real it follows from (A.1) that

$I_{K K^{\prime}}^{R}(J, m)=\int_{-1}^{+1} d(\cos \beta) d_{K K^{\prime}}^{J}(\beta) \cos m \beta$

P. Chattopadhyay

Institut für Theoretische Physik

Universität Frankfurt/Main

Robert-Mayer-Straße 8-10

D-6000 Frankfurt/Main 1

Federal Republic of Germany 